\title{
Metamorphosis of helical magnetorotational instability in the presence of axial electric current
}

\author{
Jānis Priede \\ Applied Mathematics Research Centre, Coventry University, Coventry, CV1 5FB, United Kingdon *
}

\begin{abstract}
This paper presents numerical linear stability analysis of a cylindrical Taylor-Couette flow of liquid metal carrying axial electric current in a generally helical external magnetic field. Axially symmetric disturbances are considered in the inductionless approximation corresponding to zero magnetic Prandtl number. Axial symmetry allows us to reveal an entirely new electromagnetic instability. First, we show that the electric current passing through the liquid can extend the range of helical magnetorotational instability (HMRI) indefinitely by transforming it into a purely electromagnetic instability. Two different electromagnetic instability mechanisms are identified. The first is an internal pinch-type instability, which is due to the interaction of the electric current with its own magnetic field. Axisymmetric mode of this instability requires a free-space component of the azimuthal magnetic field. When the azimuthal component of the magnetic field is purely rotational and the axial component is nonzero, a new kind of electromagnetic instability emerges. The latter, driven by the interaction of electric current with a weak collinear magnetic field in a quiescent fluid, gives rise to a steady meridional circulation coupled with azimuthal rotation.
\end{abstract}

PACS numbers: 47.20.Qr, 47.65.-d, 95.30.Lz

\section{INTRODUCTION}

Certain hydrodynamically stable rotational flows of electrically conducting fluids can turn unstable in the presence of the magnetic field. This rather counterintuitive effect was first predicted by Velikhov [1] and Chandrasekhar [2, 3] for cylindrical Taylor-Couette (TC) flow of a perfectly conducting fluid subject to axial magnetic field. After three decades of obscurity the MRI was re-discovered by Balbus and Hawley who speculated that it could account for the fast formation of stars by driving turbulent transport of angular momentum in accretion disks [4]. This hypothesis has spurred many theoretical and numerical studies [5] as well as several attempts to reproduce the MRI in the laboratory [6]. Though there is little doubt about the reality of MRI, which follows directly from classical fluid mechanics and electrodynamics, a convincing experimental demonstration of this effect is hindered by a serious technical issue. Like the magnetohydrodynamic dynamo, the MRI requires the magnetic Reynolds number $\mathrm{Rm} \sim 10$. For common liquid metals, which are relatively poor conductors characterized by low magnetic Prandtl numbers $\mathrm{Pm} \sim 10^{-5}-10^{-6}$, this translates into a large hydrodynamic Reynolds number $\mathrm{Re}=\mathrm{Rm} / \mathrm{Pm} \sim 10^{6}-10^{7}$ [7]. At this high Reynolds numbers most flows become turbulent due to inherently hydrodynamic mechanisms independent of the MRI.

A way to circumvent this technical issue was suggested by Hollerbach and Rüdiger [8], who found that the threshold of MRI in cylindrical TC flow drops to $\operatorname{Re} \sim 10^{3}$ when the imposed magnetic field is helical rather than purely axial as for the standard MRI (SMRI). This helical type of MRI (HMRI) turned out to be significantly weaker and much more limited than the SMRI [9]. Nevertheless, an instability closely resembling the HMRI was shortly observed in the PROMISE experiment [10]. Subsequent analysis revealed that this instability has been observed slightly beyond the narrow range

*J.Priede@ coventry.ac.uk in which the existence of HMRI is predicted by the ideal TC flow model [11]. This apparently small discrepancy between the theory and experiment hides two major issues pertinent to the HMRI. First, due to the hydrodynamic [12] and electromagnetic [11] end effects, the real base flow, in which the HMRI is to be observed, inevitably deviates from the ideal TC flow used by the underlying theory. The end effects can be reduced to some degree, as in the modified PROMISE experiment [13], but they cannot be eliminated completely. Although the end effects can be taken into account by realistic numerical models, which can achieve a good agreement with the experiment, this does not solve the main problem, which is the identification of the HMRI. Namely, the HMRI is physically indistinguishable from a magnetically modified hydrodynamic Taylor vortex flow. The distinction between both is only theoretical and based on the hydrodynamic stability limit. The latter is well defined only for ideal TC flow but not for a realistic base flow affected by the end effects. It is not obvious how to determine this stability limit for a real base flow affected by both hydrodynamic and magnetic end effects. Neither experiment nor direct numerical simulation is able to discriminate between the HMRI and other possible hydromagnetic of instabilities.

The second issue that makes the identification of the HMRI particularly hard is the very short extension of this instability, especially its self-sustained (absolute) mode, beyond the hydrodynamic stability limit [11]. It is the narrow confinement of the HMRI behind the hydrodynamic stability limit which makes the exact location of this limit so important for the identification of the HMRI. Besides the identification problem, the short extension of the HMRI implies a limited astrophysical relevance of this instability. Namely, though the HMRI is able to destabilize certain centrifugally stable velocity distributions, it does not reach up to the astrophysically relevant Keplerian rotation profile [9, 14, 15].

Recently, it was suggested by Kirillov and Stefani [16] that the range of HMRI can significantly be extended when the azimuthal magnetic field component is allowed to have a nonzero rotation. This apparently minor mathematical modi- 
fication of the model has several far-reaching physical consequences which are the main concern of the present paper. First , a nonpotential azimuthal magnetic field physically means the presence of axial electric current in the fluid which provides an electromagnetic energy source in addition to the mechanical rotation. As a result, instability can develop without the background flow and thus, in principle, extend over an unlimited range of velocity profiles. In this paper we show that there are two such instabilities which appear in the presence of background electric current. The first is the resistive mode of internal pinch-type instability which was originally predicted by Michael [17] in ideally conducting Taylor-Couette flow bounded by solid walls where it is expected to develop on the Alfvén time scale [1]. The second appears to be a new type of resistive instability driven by the interaction of axial electric current with a weak collinear external magnetic field.

The first type of instability presents a certain astrophysical interest as it is thought to affect the stars containing toroidal magnetic fields [18, 19]. Because the strong radial stratification in stellar interiors makes this instability nearly horizontal and thus significantly differ from other pinch-type instabilities [20], Spruit [21] termed it Tayler instability. This term was later used in a much broader sense by Rüdiger et al. [22] to refer to current-driven instabilities in homogenous fluids including liquid metals which are highly resistive from astrophysical point of view. Such a resistive instability was presumably observed in the recent liquid-metal experiment by Seilmayer et al. [23].

Although axial magnetic field has been extensively studied as a means of stabilization of the plasma pinch [24-26], its potentially destabilizing effect in the highly resistive liquids bounded by solid walls seems to have been overlooked so far. The previous studies of the pinch instability in resistive fluids have been limited to the conventional case of deformable boundaries [27]. In this case, axial magnetic field applied along a liquid metal jet carrying electric current is known to cause a kink instability [28].

The paper is organized as follows. The problem is formulated in Sec. II Numerical results for various magnetic field configurations are presented in Sec. IIII The paper is concluded with a brief discussion and summary of results in Sec. IV.

\section{FORMULATION OF THE PROBLEM}

Consider an incompressible fluid of kinematic viscosity $v$ and electrical conductivity $\sigma$ filling the gap between two infinite concentric cylinders with the inner radius $R_{i}$ and the outer radius $R_{o}$ rotating, respectively, with the angular velocities $\Omega_{i}$ and $\Omega_{o}$ in the presence of a generally helical magnetic field $\mathrm{B}_{0}=\mathrm{e}_{z} B_{z}+\mathrm{e}_{\phi} B_{\phi}$ with the axial component $B_{z}=\alpha B_{0}$ and the azimuthal component

$$
B_{\phi}=B_{0}\left[(\beta-\gamma) R_{i} / r+\gamma r / R_{i}\right]
$$

in cylindrical coordinates $(r, \phi, z)$. The dimensionless coefficient $\alpha$ defines the magnitude of axial component of the magnetic field relative to that of the azimuthal component. The

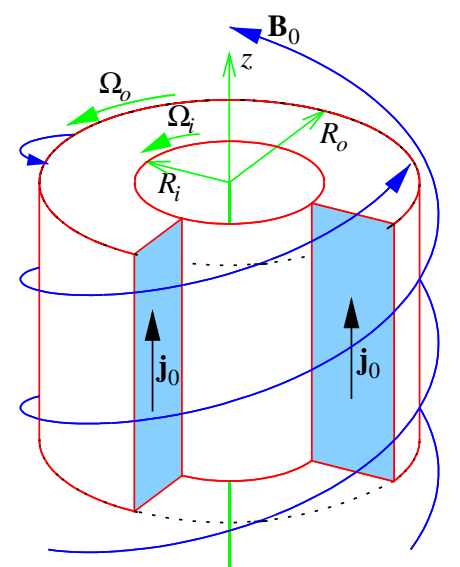

Figure 1. (Color online) Sketch of the problem.

latter has a free-space part defined by the coefficient $\beta$ and a rotational part defined by the coefficient $\gamma$, which is associated with the axial current density in the fluid $\mathrm{j}_{0}=\mu_{0}^{-1} \nabla \times \mathrm{B}_{0}=$ $\mathrm{e}_{z} \frac{2 \gamma B_{0}}{\mu_{0} R_{i}}$, where $\mu_{0}$ is the magnetic permeability of vacuum. In the annular geometry with $R_{i} \neq 0$, the absence of the current at $r<R_{i}$ produces also a free-space component of the magnetic field with the effective helicity $-\gamma$ which appears in the first term of Eq. (1). Freespace magnetic field can be modified by passing additional current along an electrode placed in the center of the annular cavity as in the PROMISE experiment [10]. This component of the magnetic field is specified by the coefficient $\beta$. Further we use $\alpha=1$ for the magnetic field with a nonzero axial component, which means $B_{0}=B_{z}$ when $B_{z} \neq 0$. A purely azimuthal magnetic field corresponds to $\alpha=0$.

Following the inductionless approximation, which holds for most liquid-metal magnetohydrodynamics characterized by small magnetic Reynolds numbers $\mathrm{Rm}=\mu_{0} \sigma v_{0} L \ll 1$, where $v_{0}$ and $L$ are the characteristic velocity and length scales, the magnetic field of the currents induced by the fluid flow is assumed to be negligible relative to the imposed field $\mathrm{B}_{0}$ everywhere except the electromagnetic force term in the NavierStokes equation

$$
\partial_{t} \mathrm{v}+(\mathrm{v} \cdot \nabla) \mathrm{v}=\rho^{-1}(-\nabla p+\mathrm{j} \times \mathrm{B})+v \nabla^{2} \mathrm{v},
$$

where, as shown below, its interaction with the background electric current $j_{0}$ results in a non-negligible perturbation of the electromagnetic body force. The electric current density is governed by Ohm's law for a moving medium,

$$
\mathrm{j}=\sigma\left(\mathrm{E}+\mathrm{v} \times \mathrm{B}_{0}\right)
$$

and related to the magnetic field by Ampère's law, $\mathrm{j}=\mu_{0}^{-1} \nabla \times$ $B$. In addition, we assume that the characteristic time of velocity variation is much longer than the magnetic diffusion time $\tau_{0} \gg \tau_{m}=\mu_{0} \sigma L^{2}$. This leads to the quasistationary approximation according to which $\nabla \times \mathrm{E}=0$ and $\mathrm{E}=-\nabla \Phi$, where $\Phi$ is the electrostatic potential. Mass and charge conservation imply $\nabla \cdot \mathrm{v}=\nabla \cdot \mathrm{j}=0$. 
The problem admits a base state with a purely azimuthal velocity distribution $\mathrm{v}_{0}(r)=\mathrm{e}_{\phi} v_{0}(r)$, where

$$
v_{0}(r)=r \frac{\Omega_{o} R_{o}^{2}-\Omega_{i} R_{i}^{2}}{R_{o}^{2}-R_{i}^{2}}+\frac{1}{r} \frac{\Omega_{o}-\Omega_{i}}{R_{o}^{-2}-R_{i}^{-2}} .
$$

Note that this base flow is not affected by the magnetic field and remains the same as in the hydrodynamic case. First, this is because the unperturbed electromagnetic force is potential, and thus can be compensated by a radial pressure gradient. Second, there is no current and thus no additional electromagnetic force generated by the base flow which gives rise only to the electrostatic potential $\Phi_{0}(r)=B_{0} \int v_{0}(r) d r$, whose gradient compensates the induced electric field. Current can appear only in the perturbed state

$$
\left\{\begin{array}{c}
\mathrm{v}, p \\
\mathrm{~B}, \Phi
\end{array}\right\}(\mathrm{r}, t)=\left\{\begin{array}{c}
\mathrm{v}_{0}, p_{0} \\
\mathrm{~B}_{0}, \Phi_{0}
\end{array}\right\}(r)+\left\{\begin{array}{c}
\mathrm{v}_{1}, p_{1} \\
\mathrm{~B}_{1}, \Phi_{1}
\end{array}\right\}(\mathrm{r}, t),
$$

where $\mathrm{v}_{1}, p_{1}, \mathrm{~B}_{1}$, and $\Phi_{1}$ are small-amplitude perturbations for which Eqs. (2 and 3) after linearization take the form

$$
\begin{aligned}
\partial_{t} \mathrm{v}_{1} & +\left(\mathrm{v}_{1} \cdot \nabla\right) \mathrm{v}_{0}+\left(\mathrm{v}_{0} \cdot \nabla\right) \mathrm{v}_{1} \\
& =\rho^{-1}\left(-\nabla p_{1}+\mathrm{j}_{1} \times \mathrm{B}_{0}+\mathrm{j}_{0} \times \mathrm{B}_{1}\right)+v \nabla^{2} \mathrm{v}_{1} \\
\mathrm{j}_{1} & =\sigma\left(-\nabla \Phi_{1}+\mathrm{v}_{1} \times \mathrm{B}_{0}\right)=\mu_{0}^{-1} \nabla \times \mathrm{B}_{1} .
\end{aligned}
$$

Taking the curl of Eq. (5) to eliminate the potential gradient we obtain the following induction equation

$$
\sigma \nabla \times\left(\mathrm{v}_{1} \times \mathrm{B}_{0}\right)+\mu_{0}^{-1} \nabla^{2} \mathrm{~B}_{1}=0
$$

The subsequent analysis is limited to axisymmetric perturbations which are not necessary the most unstable but still useful for elucidating the basic instability mechanisms. For such perturbations, the solenoidity constraints are satisfied by introducing meridional stream functions $\psi$ and $h$ for the fluid flow and electric current as

$$
\begin{gathered}
\mathrm{v}=v \mathrm{e}_{\phi}+\nabla \times\left(\psi \mathrm{e}_{\phi}\right), \\
\mathrm{j}=j \mathrm{e}_{\phi}+\nabla \times\left(h \mathrm{e}_{\phi}\right) .
\end{gathered}
$$

Note that $h$ is the azimuthal component of the induced magnetic field which is governed by Eq. (6) and used subsequently instead of $\Phi$ for the description of the induced current. Equation (4) contains not only the azimuthal current, which is explicitly related to the radial velocity, but also the radial component of the induced magnetic field, which is subsequently denoted by $g$ and governed by the radial component of Eq. (6). For numerical purposes, we introduce also the vorticity

$$
\omega=\omega \mathrm{e}_{\phi}+\nabla \times\left(v \mathrm{e}_{\phi}\right)=\nabla \times \mathrm{v}
$$

as an auxiliary variable. Perturbations are sought in the normal mode form

$$
\left\{v_{1}, \omega_{1}, \psi_{1}, h_{1}, g_{1}\right\}(\mathrm{r}, t)=\{\hat{v}, \hat{\omega}, \hat{\psi}, \hat{h}, \hat{g}\}(r) \times e^{\Gamma t+i k z},
$$

where $\Gamma$ is, in general, a generally complex growth rate and $k$ is a real wave number. Henceforth, we proceed to dimensionless variables by using $R_{i}, R_{i}^{2} / v, R_{i} \Omega_{i}, B_{0}$, and $\sigma \mu_{0} B_{0} R_{i}^{2} \Omega_{i}$ as the length, time, velocity, and the induced magnetic field scales, respectively. Nondimensional governing equations then read as

$$
\begin{aligned}
\Gamma \hat{v}= & D_{k} \hat{v}+\operatorname{Re} i k r^{-1}\left(r^{2} \Omega\right)^{\prime} \hat{\psi}+\mathrm{Ha}^{2}(i k \alpha \hat{h}+2 \gamma \hat{g}), \\
\Gamma \hat{\omega}=D_{k} \hat{\omega}+2 \operatorname{Re} i k \Omega \hat{v}+ & \quad+H a^{2} i k\left[i k \alpha \hat{\psi}-2\left((\beta-\gamma) r^{-2}+\gamma\right) \hat{h}\right], \\
0 & =D_{k} \hat{\psi}+\hat{\omega}, \\
0= & D_{k} \hat{h}+i k\left[\alpha \hat{v}-2(\beta-\gamma) r^{-2} \hat{\psi}\right], \\
0= & D_{k} \hat{g}+k^{2} \alpha \hat{\psi},
\end{aligned}
$$

where $D_{k} f \equiv r^{-1}\left(r f^{\prime}\right)^{\prime}-\left(r^{-2}+k^{2}\right) f$ and the prime stands for $\frac{\mathrm{d}}{\mathrm{d} r} ; \operatorname{Re}=R_{i}^{2} \Omega_{i} / v$ and $\mathrm{Ha}=R_{i} B_{0} \sqrt{\sigma / \rho v}$ are Reynolds and Hartmann numbers, respectively;

$$
\Omega(r)=\frac{\lambda^{-2}-\mu+r^{-2}(\mu-1)}{\lambda^{-2}-1}
$$

is the dimensionless angular velocity of the base flow defined in terms of $\lambda=R_{o} / R_{i}$ and $\mu=\Omega_{o} / \Omega_{i}$.

The boundary conditions for the hydrodynamic perturbations on the inner and outer cylinders at $r=1$ and $r=\lambda$, respectively, are $\hat{v}=\hat{\psi}=\hat{\psi}^{\prime}=0$. The boundary conditions for the electric stream function $\hat{h}$ at insulating and perfectly conducting cylinders are $\hat{h}=0$ and $(r \hat{h})^{\prime}=0$, respectively. Note that the latter case should be understood as a limit only because it implies an infinite current density in the perfectly conducting walls in the presence of a non-zero axial electric current through the liquid [29]. The effective boundary conditions for the radial component of the induced magnetic field $\hat{g}$ follow from the free-space solution of Eq. (11) with $\hat{\psi} \equiv 0$, which yields

$$
\hat{g}(r)= \begin{cases}G_{i} I_{1}(k r), & 0 \leq r \leq 1 \\ G_{o} K_{1}(k r), & r \geq \lambda,\end{cases}
$$

where $I_{1}$ and $K_{1}$ are the modified Bessel functions of the first and second types of index 1 [30]. Taking the ratio $(r \hat{g})^{\prime} / \hat{g}$ to eliminate the unknown constants $G_{i}$ and $G_{o}$, we obtain the sought boundary conditions in the form

$$
\begin{aligned}
& (r \hat{g})^{\prime}=c_{i}(k r) \hat{g} \text { at } r=1, \\
& (r \hat{g})^{\prime}=c_{o}(k r) \hat{g} \text { at } r=\lambda,
\end{aligned}
$$

where $c_{i}(r)=r I_{0}(r) / I_{1}(r)$ and $c_{o}(r)=-r K_{0}(r) / K_{1}(r)$. Because the radial magnetic field component $\hat{g}$ is generated by the azimuthal current, which is tangential to the boundaries, it is not affected by the conductivity of walls. Thus, the boundary conditions above apply to both insulating and perfectly conducting cylinders.

Equations (7)-111) were solved numerically using a spectral collocation method on a Chebyshev-Lobatto grid with a typical number of internal points $N=32$. In order to avoid spurious eigenvalues, auxiliary Dirichlet boundary conditions for $\hat{\omega}$ were introduced and then numerically eliminated using the no-slip boundary conditions $\hat{\psi}^{\prime}=0$ [31]. The electromagnetic variables $\hat{h}$ and $\hat{g}$ were represented in terms of $\hat{v}$ and $\hat{\psi}$ by numerical solution of Eqs. (10 and 11) and then substituted into Eqs. (7) and 8). The resulting standard complex matrix eigenvalue problem of the size $2 N \times 2 N$ was solved by the LAPACK ZGEEV routine. 

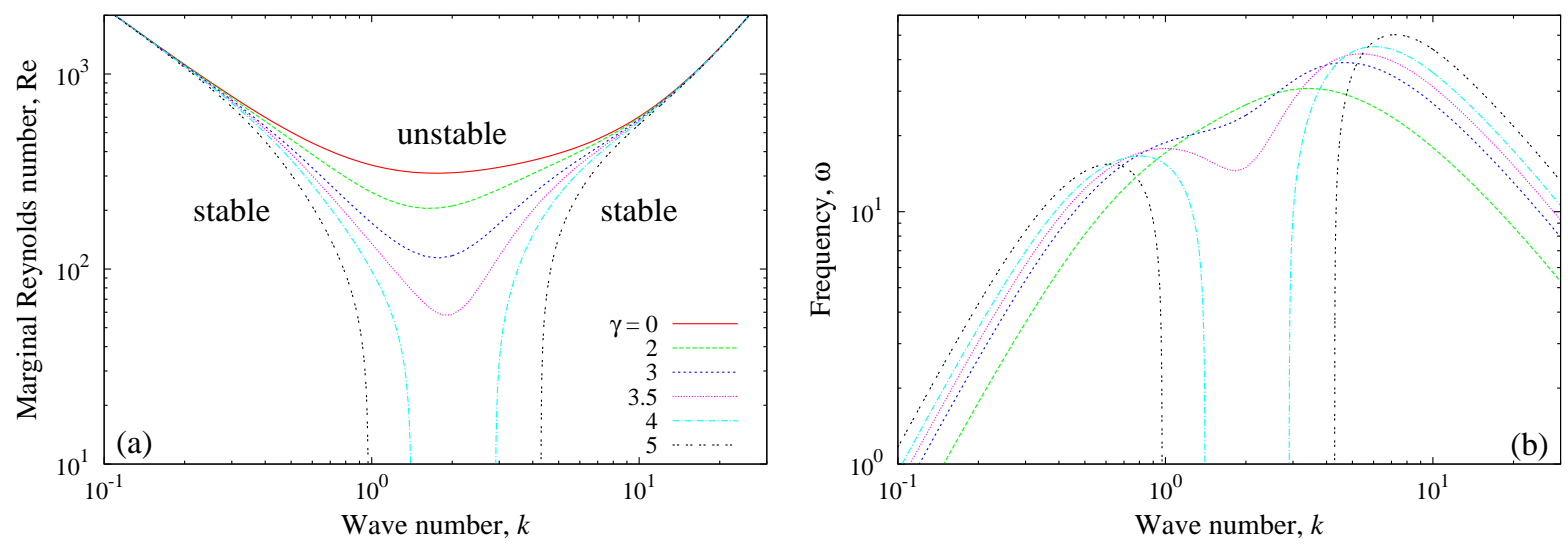

Figure 2. (Color online) Marginal Reynolds number (a) and the frequency (b) versus wave number for a hydrodynamically unstable flow with $\mu=0.2$ at various helicities $\gamma$ of rotational helical magnetic field with $\alpha=1, \beta=0$ and $\mathrm{Ha}=10$.

\section{RESULTS}

\section{A. Degeneration of the HMRI in the presence of axial electric current}

In the following, the radii ratio of inner and outer cylinders is fixed to $\lambda=2$ and the cylinders are assumed to be insulating, unless stated otherwise. We start with a hydrodynamically unstable flow corresponding to the ratio of rotation rates $\mu=0.2$, which is below the Rayleigh limit $\mu_{c}=\lambda^{-2}=0.25$. The magnetic field is helical with the axial component fixed by $\alpha=1$ and the azimuthal component generated only by the current passing through the fluid, which corresponds to $\beta=0$. In a purely axial magnetic field corresponding to $\gamma=0$, the flow becomes centrifugally unstable to stationary Taylor vortices when Reynolds number exceeds the marginal value which is plotted in Fig. 2 a) against the wave number $k$. Addition of a weak azimuthal magnetic field reduces the instability threshold and makes the instability oscillatory with the frequency $\omega=\mathfrak{I}[\Gamma]$ which is shown in Fig. 2] b). The most important result seen in Fig. 22 a) is the drop of marginal Reynolds number to zero in a range of intermediate wave numbers when the helicity of the field due the axial current defined by $\gamma$ becomes somewhat greater than 3.7. Zero Reynolds number means that this instability becomes entirely electromagnetic. Moreover, Fig. 2(b) shows that this instability is stationary, i.e., $\omega=0$. It will be shown later that two different electromagnetic mechanisms may be behind this instability.

Next, let us turn to a hydrodynamically stable case corresponding to the ratio of rotation rates set to $\mu=0.3$, which is slightly above the Rayleigh limit $\mu_{c}=0.25$. As seen in Fig. 3 (a), a moderately helical rotational magnetic field can destabilize this flow similarly to the helical free-space magnetic field [32]. In both cases neutral stability curves form closed contours, which means that the instability can occur only in limited ranges of Reynolds and wave numbers. In contrast to the hydrodynamically unstable case considered above, there are now two marginal Reynolds numbers - the lower one by exceeding which the flow destabilizes, and the upper one by exceeding which the flow restabilizes. The existence of the upper critical Reynolds number is another peculiarity of the HMRI which, in principle, distinguishes it from a magnetically modified Taylor vortex flow [32]. The upper critical Reynolds number and the associated islands of instability appear also in a centrifugally unstable regime with an axial through flow [33].

This picture changes when the helicity of the rotational field exceeds $\gamma \approx 3.7$. As for the hydrodynamically unstable case considered above, marginal Reynolds number again drops to zero in a certain range of intermediate wave numbers. Figure 4 (a) shows the critical Reynolds number and the respective frequency versus the ratio of rotation rates of inner and outer cylinders $\mu$ at various helicities $\gamma$ of rotational helical magnetic field with $\alpha=1, \beta=0$, and $\mathrm{Ha}=10$. As the axial current defined by $\gamma$ is increased, the lower critical Reynolds number reduces and the range of instability beyond the Rayleigh limit increases until the critical value $\gamma \approx 3.7$ is attained. At this critical helicity, the lower critical Reynolds number drops to zero and the range of instability becomes effectively unlimited. It is important to note that the extension of instability beyond the Rayleigh limit reduces with the increase of Reynolds number. This corresponds to the restabilization of the flow by the fast rotation, which takes place above the upper critical Reynolds number plotted in 4 (a) for the values of $\mu$ beyond the Rayleigh limit.

\section{B. Instability in the azimuthal magnetic field generated by axial current in the liquid}

Let us consider next what happens when the axial component of the magnetic field is switched off by setting $\alpha=0$. It means that the magnetic field is now perfectly azimuthal and generated only by the axial current in the liquid. Marginal Reynolds number and the frequency for both hydrodynamically unstable $(\mu=0.2)$ and stable $(\mu=0.3)$ flows in the magnetic fields of various strength defined by $\gamma$ and $\mathrm{Ha}=10$ are plotted against the wave number $k$ in Fig. 5. For the hydro- 

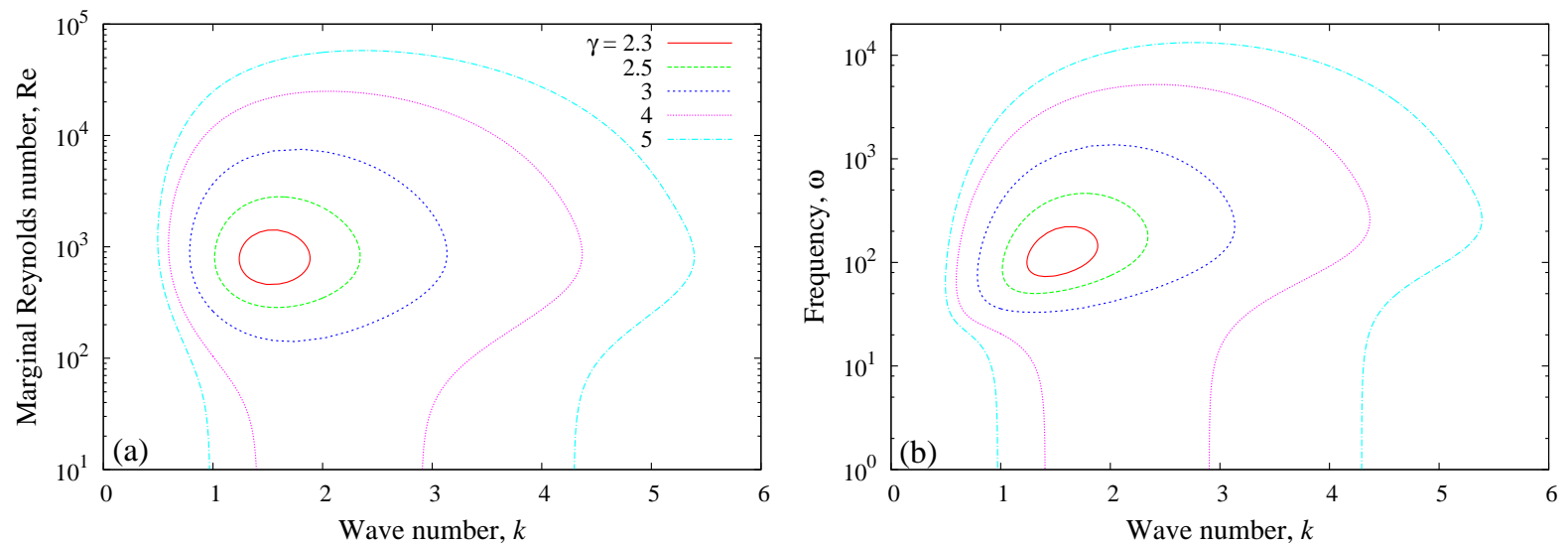

Figure 3. (Color online) Marginal Reynolds number (a) and the frequency (b) versus the wave number for a hydrodynamically stable flow with $\mu=0.3$ at various helicities $\gamma$ of rotational helical magnetic field with $\alpha=1, \beta=0$, and $\mathrm{Ha}=10$.
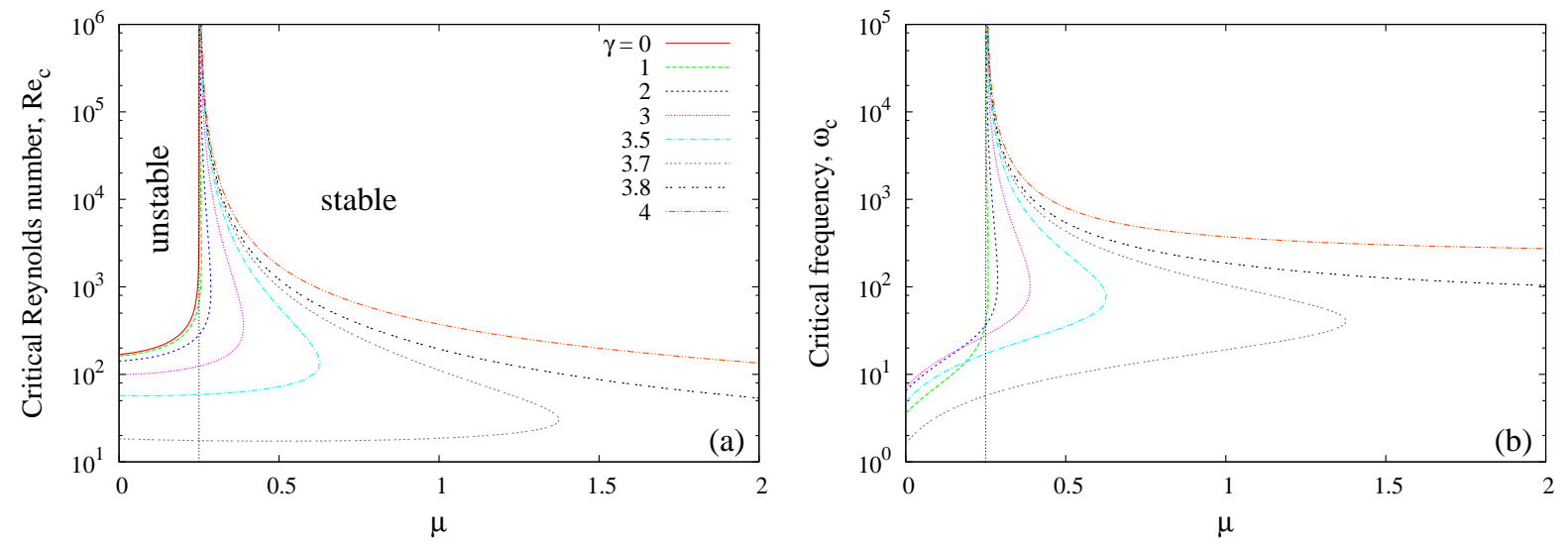

Figure 4. (Color online) Critical Reynolds number (a) and the frequency (b) versus the ratio of rotation rates of inner and outer cylinders $\mu$ at various helicities $\gamma$ of rotational helical magnetic field with $\alpha=1, \beta=0$ and $\mathrm{Ha}=10$.

dynamically unstable flow, the effect of the azimuthal field is very similar to that of the helical field considered previously. Namely, the increase of the axial electric current defined by $\gamma$ reduces marginal Reynolds number, which again drops to zero in a certain range of wave numbers when $\gamma \gtrsim 4.5$. In contrast to helical magnetic field, now the instability is completely stationary, i.e., $\omega=0$. For hydrodynamically stable flow, the effect slightly differs from that of the helical field. First, in this case all neutral stability curves, which as before exist only for a limited range of wave numbers, end at zero Reynolds number. It means that the lower critical Reynolds number, if any, is always zero when the flow is hydrodynamically stable. Second, as seen in Fig. 5, an oscillatory instability mode appears contrary to Edmonds [29] conjecture in a certain subrange of unstable wave numbers at sufficiently high Re when $\gamma \gtrsim 6$. This oscillatory mode, which resembles an electromagnetically destabilized inertial wave, persists up to much higher Reynolds numbers than the stationary one.

The stationary mode looks like a pinch-type instability which has been studied in this setup numerically by Shalybkov using a more general non-axisymmetric and finite- $\mathrm{Pm}$

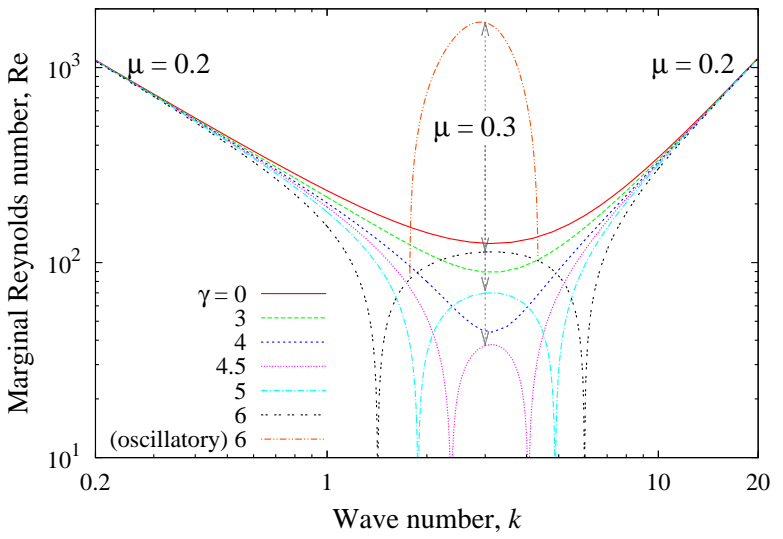

Figure 5. (Color online) Marginal Reynolds number versus wave number for hydrodynamically unstable $(\mu=0.2)$ and stable $(\mu=$ $0.3)$ flows in the azimuthal magnetic field $(\alpha=0)$ generated only by the axial current in the liquid annulus $(\beta=0)$ with various magnitude $\gamma$ at $\mathrm{Ha}=10$. 


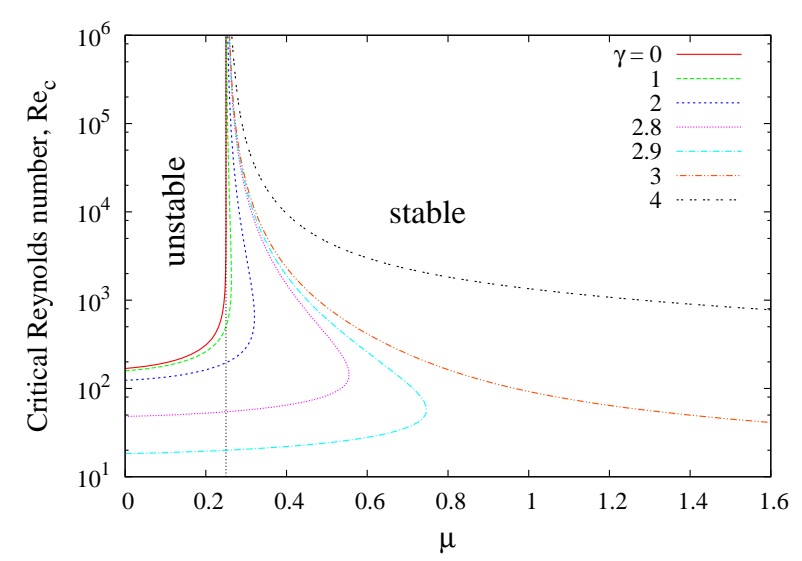

Figure 6. (Color online) Critical Reynolds number versus the ratio of rotation rates of inner and outer cylinders $\mu$ at various helicities $\gamma$ of purely rotational helical magnetic field with $\alpha=1, \beta=\gamma$, and $\mathrm{Ha}=10$.

approximation [34, 35] and Rüdiger et al. [22] in the context of the so-called azimuthal MRI. The latter is inherently nonaxisymmetric [36] and has the same limited extension beyond the Rayleigh line as the HMRI in the highly resistive limit [15]. Undeterred by the associated identification challenges, which we discussed in the introduction, Seilmayer et al. [37] claim to have observed this instability in another recent liquidmetal experiment.

Pinch-type instability operates through the compression of the azimuthal magnetic field lines by a radially inward flow perturbation which amplifies itself by enhancing the electromagnetic pinch force generated by the interaction of the axial electric current with its own magnetic field. It is important to notice that axisymmetric meridional flow interacts only with the free- space $\left(\sim r^{-1}\right)$ but not with the rotational $(\sim r)$ component of the azimuthal magnetic field [17, 29]. As is easy to see from Eq. (10), the respective induction term proportional to $\beta-\gamma$ is entirely due to the free-space component of the magnetic field, and vanishes together with the latter when $\gamma=\beta$. The interaction between axisymmetric meridional flow and azimuthal rotational magnetic field is precluded by the conservation of the magnetic flux. The flux is conserved because the rotational magnetic field varies linearly with the cylindrical radius $r$ while the respective cross-sectional area of a toroidal element of constant volume in incompressible fluid flow varies inversely with $r$. Thus, in contrast to the conventional $z$ pinch, this instability requires not only a rotational but also a free-space component of the azimuthal magnetic field. The latter, however, is possible only in annular but not in cylindrical geometry. As seen from Eq. (1), the free-space component of the azimuthal magnetic field associated to the axial electric current in annular geometry $\left(R_{i} \neq 0\right)$ can be compensated by an additional free-space magnetic field with $\beta=\gamma$, which leaves only the rotational component $\sim r$ as in the solid cylinder.

\section{Instability in helical magnetic field}
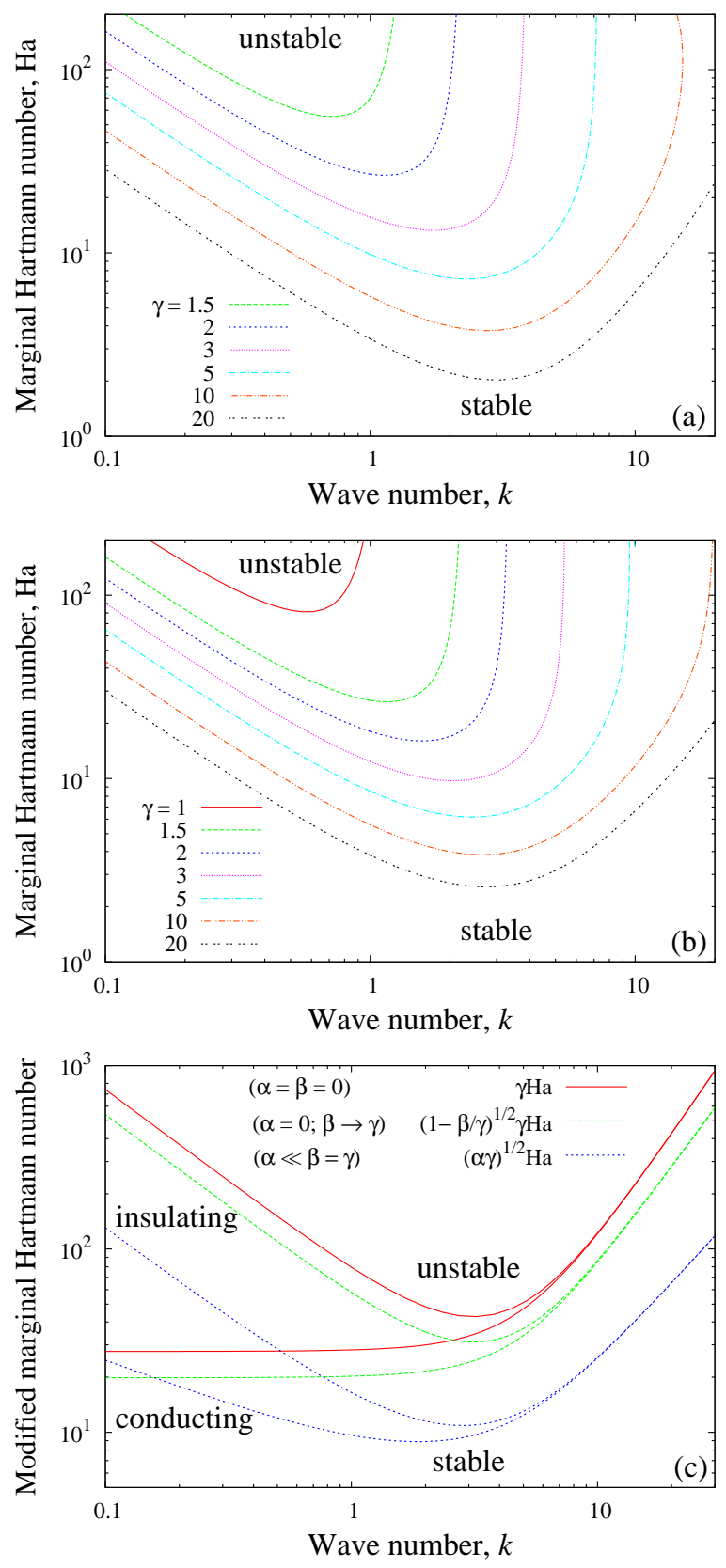

Figure 7. (Color online) Marginal Hartmann number versus wave number for purely electromagnetic $(\operatorname{Re}=0)$ stationary $(\omega=0)$ instabilities in the rotational magnetic field with $\alpha=1, \beta=0$ (a), $\alpha=1, \beta=\gamma(\mathrm{b})$, and $\alpha=0, \beta=0$, and $\beta \rightarrow \gamma(\mathrm{c})$ for both insulating and perfectly conducting cylinders at various axial currents defined by $\gamma$.

with a perfectly rotational azimuthal component

Now let us check what happens when the axisymmetric pinch instability is excluded by applying a compensating freespace magnetic field with $\beta=\gamma$ which makes the azimuthal component of the magnetic field perfectly rotational, that is, purely linear in $r$. In order to have any electromagnetic effect 
on the axisymmetric disturbances, we need to add an axial magnetic field by setting $\alpha=1$. Both the critical Reynolds number and the frequency, which are shown in Fig. 6 versus the ratio of rotation rates of outer and inner cylinders for $\mathrm{Ha}=10$, look very similar to the respective characteristics shown in Fig. 4(a) for the rotational helical magnetic field with an uncompensated free-space component. As before, the increase of the axial current reduces the critical Reynolds number, which in this case drops to zero at the critical value $\beta=\gamma \approx 2.9$, leading to an unlimited extension of the instability beyond the Rayleigh limit for larger values of $\gamma$. Thus, the elimination of the pinch-type instability has a surprisingly little effect on the remaining instability.

\section{Purely electromagnetic instabilities}

Zero marginal Reynolds number means that the instability no longer depends on the background flow and is driven entirely by the electromagnetic force which is defined by Hartmann number. Marginal Ha for such electromagnetically sustained disturbances is plotted in Fig. 7 against wave number for various axial current parameters $\gamma$ in helical magnetic field with uncompensated $(\alpha=1, \beta=0)$ (a) and compensated $\beta=\gamma$ (b) free-space azimuthal components as well as in a purely azimuthal field $(\alpha=0)$ generated only by the axial current in the liquid $(\beta=0)$, and with nearly compensated freespace component $(\beta \rightarrow \gamma)$ (c). For the first two helical field configurations, marginal $\mathrm{Ha}$ is seen to vary with $\gamma$ in a similar way. For purely azimuthal field configuration, the pinch-type instability driven only by the current passing through the liquid is determined by the effective Hartmann number $\gamma \mathrm{Ha}$. As seen in Fig. [7(c), the lowest value $\gamma \mathrm{Ha}_{c} \approx 42.74$ for insulating cylinders is attained at the critical wave number $k_{c} \approx 3.13$. When the cylinders are perfectly conducting and thus the induced currents can freely close through them, the instability threshold is seen to decrease with the wave length so the lowest value $\gamma \mathrm{Ha}_{c} \approx 26.6$ is attained asymptotically at $k \rightarrow 0$. This instability gives rise to a steady meridional flow whose streamlines and the associated electric current lines for insulating boundaries are shown in Fig. 8 .

Critical Hartmann and wave numbers for all three basic field configurations are summarized in Fig. 9 for both insulating and perfectly conducting boundaries. It is seen that at a sufficiently large $\gamma$, the instability in helical magnetic field with a nonzero (uncompensated) free-space azimuthal component turns into the pinch instability with $\mathrm{Ha}_{c} \sim \gamma^{-1}$. When this pinch-type instability is excluded by setting $\beta=\gamma$, which corresponds to a compensated free-space azimuthal component of the magnetic field, the critical Hartmann number at large $\gamma$ varies differently as $\mathrm{Ha}_{c} \sim \gamma^{-1 / 2}$. This implies a different type of instability which is driven by the interaction of axial electric current with a collinear external magnetic field. As seen in Fig. 7(c), in contrast to the pinch-type instability, this instability has a finite critical wave length not only for insulating but also for perfectly conducting cylinders. The latter fact implies that this instability relies on the closure of induced currents within the liquid which is discussed in more
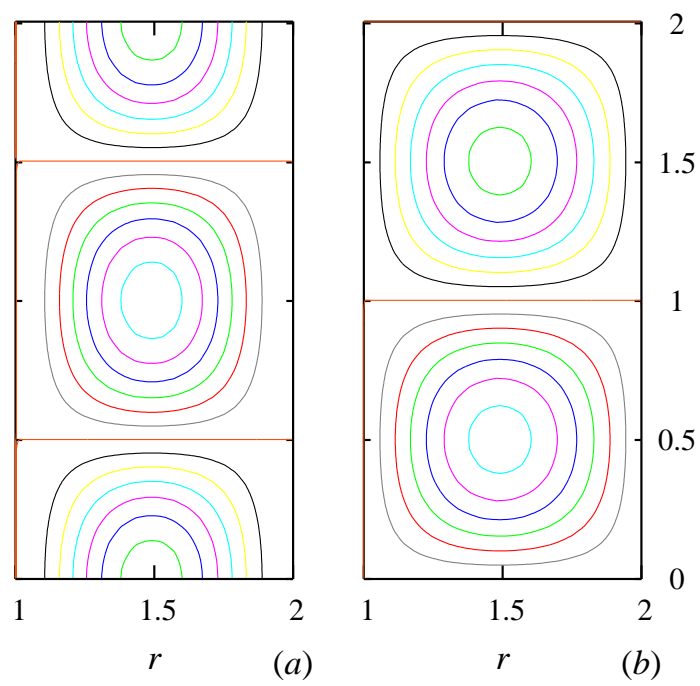
$z$

Figure 8. (Color online) Streamlines (a) and the electric current lines (a) of the critical perturbation for the electromagnetic $(\operatorname{Re}=0)$ pinch-type instability $(\alpha=\beta=0)$.

detail in the concluding section. The critical perturbation pattern of this rather complex instability for insulating cylinders is shown in Fig. 10.

Figure 11 shows the critical Hartmann number based on the gap width against the inner radius of the annular gap for both the pinch-type instability $(\alpha=\beta=0)$ and the instability driven by the electric current in a weak axial magnetic field $(\alpha \ll \beta=\gamma)$. In the limits $R_{i} \rightarrow 0$ and $R_{i} \rightarrow \infty$, the annular layer turns into a cylinder and a flat layer, respectively. Note that according to our parametrization of the magnetic field (1), the axial current density diverges as $\gamma / R_{i} \rightarrow \infty$ when $R_{i} \rightarrow 0$. The critical Hartmann number for the pinch-type instability based on this singular current density approaches a constant value when $R_{i} \rightarrow 0$ and to increases as $\sim R_{i}^{3 / 2}$ for $R_{i} \gg 1$. The critical Hartmann number based on the fixed current density, i.e., rescaled with $R_{i}^{-1}$, which is plotted in Fig. 11, attains minima at $R_{i} \approx 1$ and increases as $\sim R_{i}^{-1}$ and $\sim R_{i}^{1 / 2}$ for $R_{i} \ll 1$ and $\gg 1$, respectively. It means that, the related pinch instability vanishes not only in the cylindrical geometry, where $\beta=\gamma$, but also in the planar unbounded layer, where the associated electromagnetic force can be shown become purely irrotational. The critical Hartmann number rescaled with the current density for the other instability is seen to remain finite in both limits of $R_{i}$. It means that in contrast to the pinch instability, this instability has a finite critical current density also in the cylindrical geometry. However, despite the finite critical Hartmann number for $R_{i} \rightarrow \infty$, this instability has no analog in the planar unbounded layer. In this case, critical Hartmann number remains finite when $R_{i} \rightarrow \infty$ because the azimuthal magnetic field (1) for $\beta=\gamma$ diverges as $\sim R_{i}$. When the current density is rescaled by $1 / R_{i}$ to have a finite magnetic field when $R_{i} \rightarrow \infty$, the critical Hartmann number for $R_{i} \gg 1$ also increases as $\sim R_{i}^{1 / 2}$.

Finally, let us consider the effect of additional free-space 

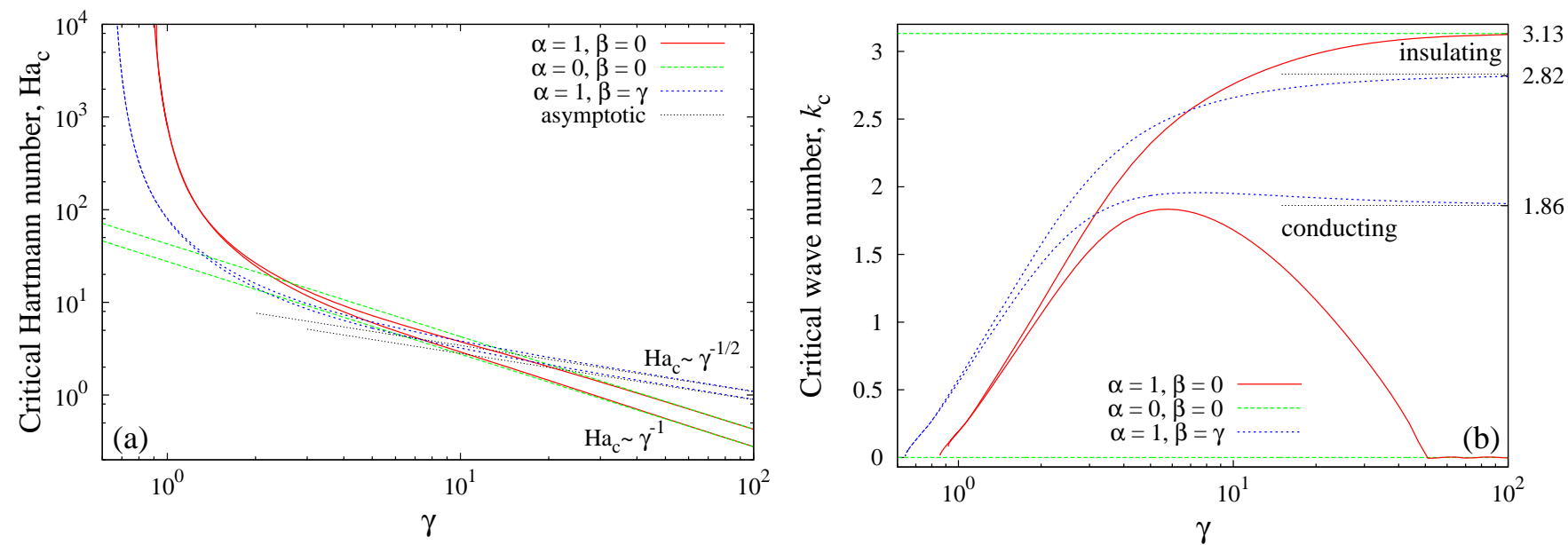

Figure 9. (Color online) Critical Hartmann number (a) and wave number (b) for purely electromagnetic $(\operatorname{Re}=0)$ stationary $(\omega=0)$ instabilities versus the axial current parameter $\gamma$ for different magnetic field configurations. The upper and lower branches for each configuration correspond to insulating and perfectly conducting cylinders.
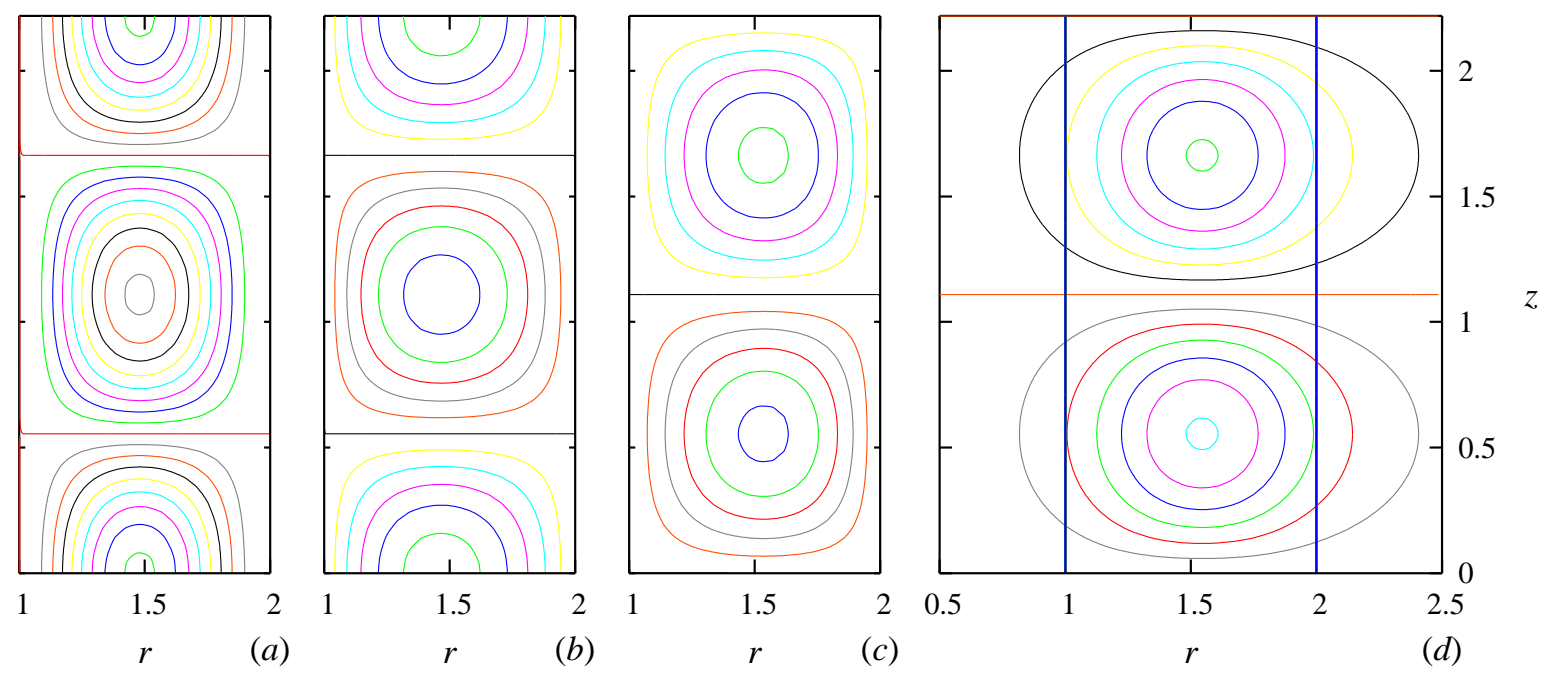

Figure 10. (Color online) Streamlines (a), isolines of the azimuthal velocity (b), the electric current lines (c), and the meridional magnetic flux lines (d) for the critical perturbation of electromagnetic $(\operatorname{Re}=0)$ rotational instability in the asymptotic case $\alpha \ll \beta=\gamma$.

azimuthal field on the pinch-type instability without axial magnetic field $(\alpha=0)$. Critical Hartmann number and the wave numbers for this case are shown in Fig. 12 versus $1-\beta / \gamma$, which defines the relative strength of the free-space component. As seen in Fig. 12, the critical Hartmann number attains the minimum $\mathrm{Ha}_{c} \approx 42.74 \gamma^{-1}$ at $\beta / \gamma$ slightly less than 0 and increases asymptotically as $\mathrm{Ha}_{c} \sim 31 \gamma^{-1}(1-\beta / \gamma)^{-1 / 2}$ when $\beta / \gamma \rightarrow 1$. Marginal Hartmann number for this limit, which corresponds to a nearly compensated free-space azimuthal component of the magnetic field, is plotted in Fig. 7(c). Asymptotic result is obtained by dropping the term with $\beta-\gamma$ in Eq. (8) which produces a quadratically small effect relative to analogous term in Eq. (8) when $\beta \rightarrow \gamma$. Critical Hartmann number becomes very large also when $\beta / \gamma \rightarrow-2$, which corresponds a compensated total axial current through the system. In this case, the current which passes through the liquid returns along a central electrode and thus cancels the field in the free space outside the system. This setup has been suggested by Stefani et al. [38] as a possible means of avoiding pinch-type instability in the future liquid metal batteries.

\section{SUMMARY AND CONCLUSIONS}

The present study was concerned with numerical linear stability analysis of a cylindrical Taylor-Couette flow of liquid metal carrying an axial electric current in the presence of a generally helical external magnetic field. It was shown that the electric current passing through the liquid profoundly alters the nature of the helical MRI by transforming it into a 


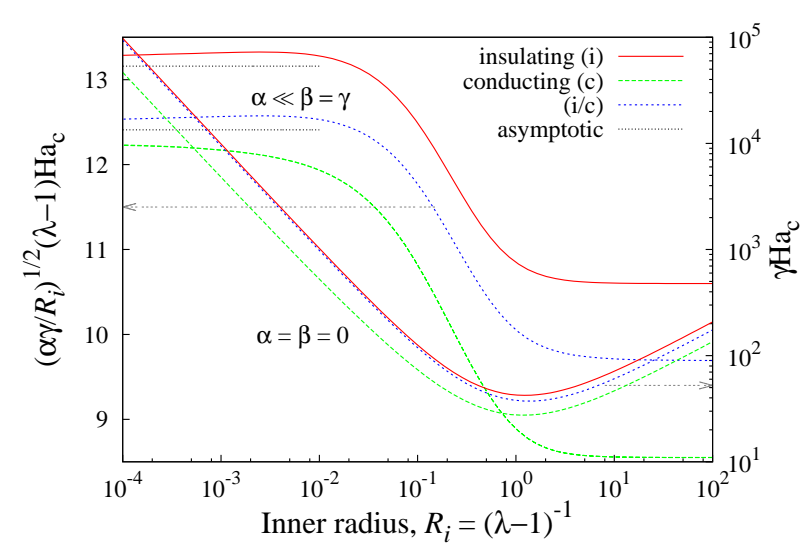

Figure 11. (Color online) Critical Hartmann number rescaled with the gap width $\lambda-1$ versus the rescaled inner radius $R_{i}=(\lambda-1)^{-1}$ for the pinch instability $(\alpha=\beta=0)$ and for the instability driven by the electric current in a weak axial magnetic field $(\alpha \ll \beta=\gamma)$ with both cylinders insulating (i), perfectly conducting (c), inner cylinder insulating and outer cylinder perfectly conducting (i/c). The Hartmann number for the latter instability (on the left axis) is additionally rescaled with the effective current density $\gamma / R_{i}$.

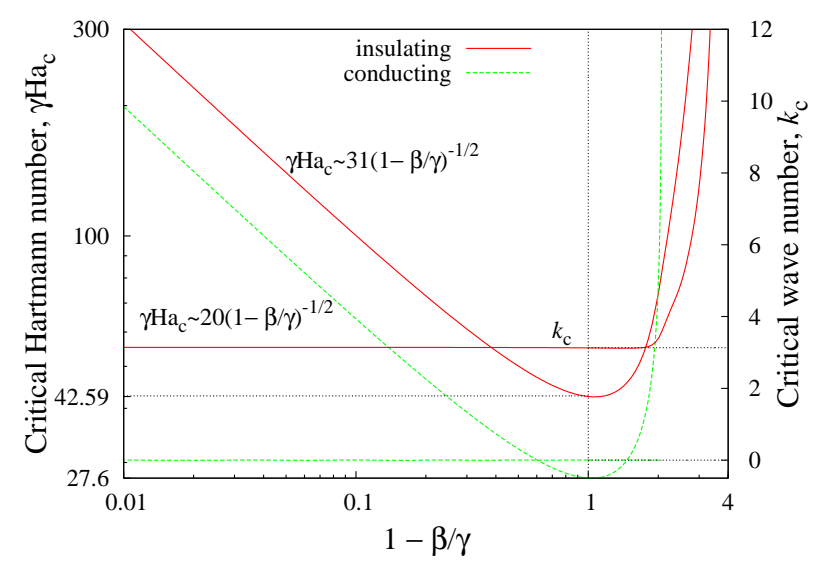

Figure 12. (Color online) Critical Hartmann number and the wave number for the electromagnetic pinch instability versus $1-\beta / \gamma$ which defines the strength of free-space azimuthal component the magnetic field relative to the rotational component generated by axial current.

purely electromagnetic instability. Two different electromagnetic instability mechanisms were identified. The first is an internal pinch-type instability which is driven by the interaction of the electric current with its own magnetic field. The axisymmetric mode of this instability considered in the present study requires a free-space component of the azimuthal mag- netic field, which is possible in the annular but not in the cylindrical geometry. In the annular geometry this instability mode can be eliminated by passing an additional current along the axis of the system to compensate the free-space azimuthal component of the magnetic field in the liquid. In this case, the addition of axial magnetic field was found to give rise to a new kind of electromagnetic instability.

The mechanism of this instability, which is driven by the interaction of axial electric current with a weak collinear external magnetic field, is as follows. First, a radially outward initial flow perturbation slightly bends the axial magnetic field but does not affect, as argued above, the purely rotational azimuthal field and the associated axial current. The deflected axial field crossing the unperturbed axial current gives rise to an azimuthal electromagnetic force which, in turn, drives an azimuthal flow perturbation. The fluid rotates in the positive direction below the radial flow perturbation, where the axial field is bent outwards, and in the negative direction above it, where the axial field bends back. Next, the azimuthal flow perturbation in the axial magnetic field induces radially outward and inward electric currents below and above the initial radial flow perturbation, respectively. These two opposite radial electric currents close in the inner part of the liquid annulus via a downward axial current, which, in turn, interacts with the azimuthal magnetic field and generates a radially outward electromagnetic force perturbation. The latter amplifies the initial radial flow perturbation, so promoting the instability.

In contrast to the azimuthal MRI [22], the helical MRI does not separate from purely electromagnetic instabilities in the inductionless limit $\mathrm{Pm}=0$. It is also important to note that although electromagnetic instabilities can develop without mechanical rotation, the latter has a stabilizing effect when the base flow is hydrodynamically stable. Similarly to the HMRI, the electromagnetic instabilities are constrained beyond the Rayleigh line to sufficiently low Reynolds numbers. This dynamical constraint may severely limit astrophysical relevance of electromagnetic instabilities. Nevertheless, there are several industrial applications such as, for example, aluminum reductions cells [39] and the prospective liquid metal batteries [40], where the strong electric current passing through the liquid metal in the presence of a collinear magnetic field can give rise to the electromagnetic instability identified in this study.

\section{ACKNOWLEDGMENTS}

This work was supported by Helmholtz Association of German Research Centres (HGF) in the framework of the LIMTECH Alliance through an agreement between Coventry University and Helmholz-Zentrum Dresden-Rossendorf.
[1] E. P. Velikhov, "Stability of an ideally conducting liquid flowing between rotating cylinders in a magnetic field," Sov. Phys. JETP 9, 995-998 (1959).

[2] S. Chandrasekhar, "The stability of non-dissipative Couette flow in hydromagnetics," Proc. Natl. Acad. Sci. USA 46, 253 257 (1960).

[3] S. Chandrasekhar, Hydrodynamic and Hydromagnetic Stability (Oxford University Press, London, 1961). 
[4] S. A. Balbus and J. F. Hawley, "A powerful local shear instability in weakly magnetized disks," Astrophys. J. 376, 214-233 (1991).

[5] S. A. Balbus and J. F. Hawley, "Instability, turbulence, and enhanced transport in accretion disks," Rev. Mod. Phys. 70, 1 (1998).

[6] H. Ji and S. Balbus, "Angular momentum transport in astrophysics and in the lab," Phys. Today 66, 27-33 (2013).

[7] J. Goodman and H. Ji, "Magnetorotational instability of dissipative Couette flow," J. Fluid Mech. 462, 365-382 (2002).

[8] R. Hollerbach and G. Rüdiger, "New type of magnetorotational instability in cylindrical Taylor-Couette flow," Phys. Rev. Lett. 95, 124501 (2005).

[9] W. Liu, J. Goodman, I. Herron, and H. Ji, "Helical magnetorotational instability in magnetized Taylor-Couette flow," Phys. Rev. E 74, 056302 (2006).

[10] F. Stefani, Th. Gundrum, G. Gerbeth, G. Rüdiger, M. Schultz, J. Szklarski, and R. Hollerbach, "Experimental evidence for magnetorotational instability in a Taylor-Couette flow under the influence of a helical magnetic field," Phys. Rev. Lett. 97, 184502 (2006).

[11] J. Priede and G. Gerbeth, "Absolute versus convective helical magnetorotational instability in a Taylor-Couette flow," Phys. Rev. E 79, 046310 (2009).

[12] F. Stefani, G. Gerbeth, Th. Gundrum, J. Szklarski, G. Rüdiger, and R. Hollerbach, "Results of a modified PROMISE experiment," Astron. Nachr. 329, 652-658 (2008).

[13] F. Stefani, G. Gerbeth, Th. Gundrum, R. Hollerbach, J. Priede, G. Rüdiger, and J. Szklarski, "Helical magnetorotational instability in a Taylor-Couette flow with strongly reduced Ekman pumping,” Phys. Rev. E 80, 066303 (2009).

[14] J. Priede, "Inviscid helical magnetorotational instability in cylindrical Taylor-Couette flow," Phys. Rev. E 84, 066314 (2011).

[15] O. N. Kirillov, F. Stefani, and Y. Fukumoto, "A unifying picture of helical and azimuthal magnetorotational instability, and the universal significance of the Liu limit," Astrophys. J. 756, 8388 (2012).

[16] O. N. Kirillov and F. Stefani, "Extending the range of the inductionless magnetorotational instability," Phys. Rev. Lett. 111, 061103 (2013).

[17] D. H. Michael, "The stability of an incompressible electrically conducting fluid rotating about an axis when current flows parallel to the axis," Mathematika 1, 45-50 (1954).

[18] Yu. V. Vandakurov, "Theory for the stability of a star with a toroidal magnetic field," Sov. Astron. 16, 265-272 (1972).

[19] R. J. Tayler, "The adiabatic stability of stars containing magnetic fields-I: Toroidal fields," Mon. Not. R. Astron. Soc. 161, 365-380 (1973).

[20] R. J. Tayler, "Hydromagnetic instabilities of an ideally conducting fluid," Proc. Phys. Soc. B 70, 31-48 (1957).

[21] H. C. Spruit, "Differential rotation and magnetic fields in stellar interiors," Astron. Astrophys. 349, 189-202 (1999).

[22] G. Rüdiger, R. Hollerbach, M. Schultz, and D. Elstner, "Destabilization of hydrodynamically stable rotation laws by azimuthal magnetic fields," Mon. Not. R. Astron. Soc. 377, 14811487 (2007).
[23] M. Seilmayer, F. Stefani, Th. Gundrum, T. Weier, G. Gerbeth, M. Gellert, and G. Rüdiger, "Experimental evidence for a transient Tayler instability in a cylindrical liquid-metal column," Phys. Rev. Lett. 108, 244501 (2012).

[24] V. D. Shafranov, "The stability of a cylindrical gaseous conductor in a magnetic field," Soviet J. Atomic Energy. 1, 709-713 (1956).

[25] W. A. Newcomb, "Hydromagnetic stability of a diffuse linear pinch,” Ann. Phys. 10, 232-267 (1960).

[26] D. D. Holm, J. E. Marsden, T. Ratiu, and A. Weinstein, "Nonlinear stability of fluid and plasma equilibria," Phys. Rep. 123, 1-116 (1985).

[27] R. J. Tayler, "Stability of twisted magnetic fields in a fluid of finite electrical conductivity," Rev. Mod. Phys. 32, 907-913 (1960).

[28] G. S. Murty, "Instability of a conducting fluid cylinder in the presence of an axial current, a longitudinal magnetic field and a coaxial conducting cylinder," Arkiv Fysik 19, 483-497 (1961).

[29] F. N. Edmonds, "Hydromagnetic stability of a conducting fluid in a circular magnetic field," Phys. Fluids 1, 30-41 (1958).

[30] M. Abramowitz and I. A. Stegun, Handbook of Mathematical Functions (Dover, New York, 1972).

[31] J. Hagan and J. Priede, "Capacitance matrix technique for avoiding spurious eigenmodes in the solution of hydrodynamic stability problems by Chebyshev collocation method,' J. Comput. Phys. 238, 210-216 (2013).

[32] J. Priede, I. Grants, and G. Gerbeth, "Inductionless magnetorotational instability in a Taylor-Couette flow with a helical magnetic field," Phys. Rev. E 75, 47303 (2007).

[33] S. Altmeyer, Ch. Hoffmann, and M. Lücke, "Islands of instability for growth of spiral vortices in the Taylor-Couette system with and without axial through flow," Phys. Rev. E 84, 046308 (2011).

[34] D. Shalybkov, "Pinch instabilities in Taylor-Couette flow," Phys. Rev. E 73, 016302 (2006).

[35] D. Shalybkov, "Rotational stabilization of pinch instabilities in Taylor-Couette flow," Phys. Rev. E 75, 047302 (2007).

[36] R. Hollerbach, V. Teeluck, and G. Rüdiger, "Nonaxisymmetric magnetorotational instabilities in cylindrical Taylor-Couette flow," Phys. Rev. Lett. 104, 044502 (2010).

[37] M. Seilmayer, V. Galindo, G. Gerbeth, Th. Gundrum, F. Stefani, M. Gellert, G. Rüdiger, M. Schultz, and R. Hollerbach, "Experimental evidence for nonaxisymmetric magnetorotational instability in a rotating liquid metal exposed to an azimuthal magnetic field," Phys. Rev. Lett. 113, 024505 (2014).

[38] F. Stefani, T. Weier, Th. Gundrum, and G. Gerbeth, "How to circumvent the size limitation of liquid metal batteries due to the Tayler instability," Energ. Convers. Manage. 52, 2982-2986 (2011).

[39] A. Pedchenko, S. Molokov, J. Priede, A. Lukyanov, and P. J. Thomas, "Experimental model of the interfacial instability in aluminium reduction cells," Europhys. Lett. 88, 24001 (2009).

[40] K. Wang, K. Jiang, B. Chung, T. Ouchi, P. J. Burke, D. A. Boysen, D. J. Bradwell, H. Kim, U. Muecke, and D. R. Sadoway, "Lithium-antimony-lead liquid metal battery for grid-level energy storage," Nature 514, 348-350 (2014). 